\title{
Staphylococcus lugdunensis Infections of the Skin and Soft Tissue: A Case Series and Review
}

Lucas A. Heldt Manica · Philip R. Cohen

Received: August 1, 2017 / Published online: October 11, 2017

(C) The Author(s) 2017. This article is an open access publication

\section{ABSTRACT}

Introduction: Staphylococcus lugdunensis (S. lugdunensis) is a coagulase-negative, Gram-positive bacterium that can be isolated as a component of normal skin flora in humans. However, more recently, it has also been documented as a culprit in skin and soft tissue infections. We describe the clinical features of five individuals with $S$. lugdunensis-associated skin infections. We review the characteristics of other patients that were previously described with this organism as the causative agent of skin infection.

Methods: Staphylococcus lugdunensis was correlated with the development of significant skin and soft tissue infections in five patients. The Pubmed database was used to search for the following terms: "abscess," "cellulitis," "cutaneous," "lugdunensis," "paronychia," "skin," "soft," "staphylococcus," and "tissue." The

Enhanced content To view enhanced content for this article go to http://www.medengine.com/Redeem/ 31BCF06046EA9476.

L. A. Heldt Manica ( $\square)$

John A. Burns School of Medicine, University of

Hawaii, Honolulu, HI, USA

e-mail: manica@hawaii.edu

P. R. Cohen $(\varangle)$

Department of Dermatology, University of

California San Diego, La Jolla, CA, USA

e-mail: mitehead@gmail.com relevant and reference papers generated by the search were reviewed.

Results: One woman and four men developed $S$. lugdunensis-related skin infections from February 19, 2015 to May 30, 2017. The patients' ages at the onset of the infection ranged from 30 to 82 years; the median age was 70 years. Four patients were older than 65 years. The back was the most common location for the infection, followed by digits. The infection presented as cystic lesions with cellulitis or periungual abscesses. The lesions were incised or spontaneously ruptured. Patients were empirically treated with oral antibiotics; if necessary, the management was adjusted based on the culture-derived sensitivities of the organisms. The infections resolved within 10-30 days after commencing treatment.

Conclusion: Staphylococcus lugdunensis has previously been considered as a nonpathogenic organism and to be a component of normal skin flora. However, S. lugdunensis can result in significant skin and soft tissue infections, perhaps more frequently in older individuals. Its antibiotic sensitivities appear to be similar to those of methicillin-susceptible Staphylococcus aureus.

Keywords: Abscess; Cellulitis; Cutaneous; Lugdunensis; Paronychia; Skin; Soft; Staphylococcus; Tissue 


\section{INTRODUCTION}

Staphylococcus lugdunensis (S. lugdunensis) is a coagulase-negative staphylococcus organism. It is typically considered to be a component of the normal flora of the skin. However, more recently, it has also been identified as a pathogen in a variety of infections, particularly in skin and soft tissue [1]. We describe five patients who developed significant skin and soft tissue infections due to $S$. lugdunensis and review their clinical features and treatments.

\section{CASE SERIES}

\section{Case 1}

A 70-year-old woman presented for evaluation of a tender lesion on her left lower back in April 2017 (Fig. 1). There was no fever or associated systemic symptoms. Her past medical history was significant for basal cell carcinoma, melanoma in situ, and seronegative rheumatoid arthritis. She was currently being treated with methotrexate.

Nine days prior to presentation, she was at the beach and felt her back being bitten by an insect. The affected area became red and painful and increased in size over the next few days.

Cutaneous examination showed an erythematous patch - the presumed bite site-with an underlying cystic component on the left lower back (Fig. 2). A small amount of pus was expressed from the lesion and cultured. The bacterial culture grew S. lugdunensis (Table 1) and antibiotic susceptibility testing was performed (Table 2).

She was treated with incision and drainage; empiric treatment with cephalexin $500 \mathrm{mg}$, four times daily for 10 days, was also initiated. A wound check performed 5 days later did not show any significant improvement. Therefore, doxycycline $100 \mathrm{mg}$, twice daily for 10 days, was added. The infection resolved during the subsequent 3 weeks. There was no recurrence of the infection within 60 days following treatment.

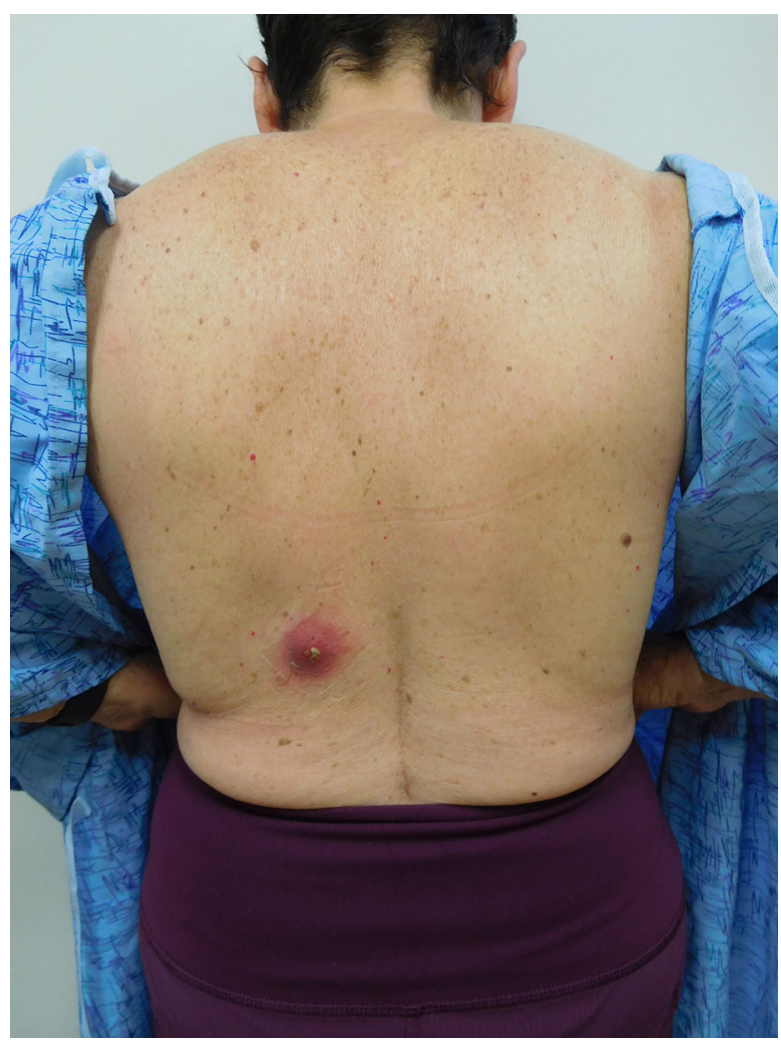

Fig. 1 Distant view of Staphylococcus lugdunensis skin and soft tissue infection presenting as a tender cystic lesion with surrounding cellulitis on the left lower back of a 70-year-old woman

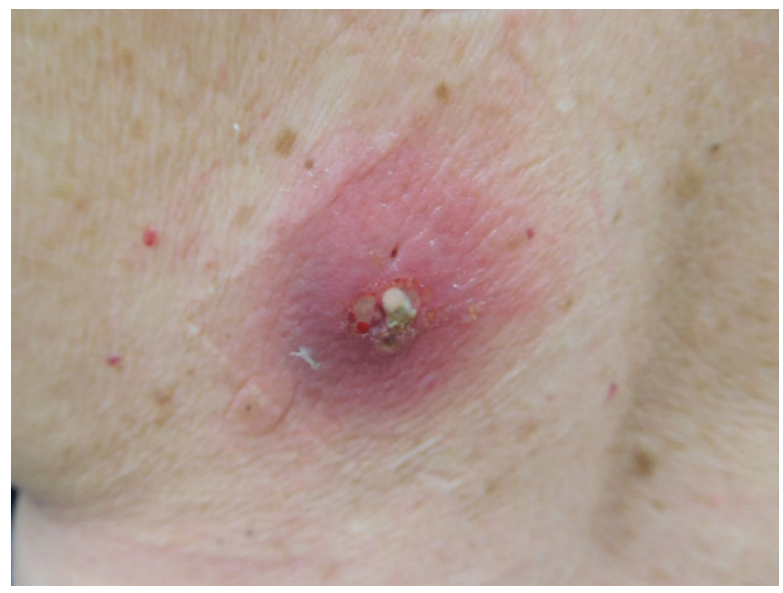

Fig. 2 Closer view of Staphylococcus lugdunensis cutaneous infection of the back of a 70-year-old woman; the infection occurred at a presumed bite site 
Table 1 Characteristics of patients with $S$. lugdunensis infections

\begin{tabular}{|c|c|c|c|c|c|}
\hline Case & $\begin{array}{l}\text { Age } \\
\text { Sex }\end{array}$ & Location & Morphology & Bacterial culture & Treatment \\
\hline 1 & $\begin{array}{l}70 \text { years } \\
\mathrm{W}\end{array}$ & Left lower back & Inflamed cyst & S. lugdunensis & $\begin{array}{l}\text { 1-Cephalexin } 500 \mathrm{mg} \text { QID for } 10 \text { days, then } \\
\text { 2-Five days later, doxycycline } 100 \mathrm{mg} \text { BID for } \\
10 \text { days was added to the antibiotic regimen }\end{array}$ \\
\hline 2 & $\begin{array}{l}30 \text { years } \\
M\end{array}$ & $\begin{array}{l}\text { Right great toe } \\
\text { nail fold }\end{array}$ & Abscess & $\begin{array}{l}1-S . \text { lugdunensis } \\
2-S . \text { aureus } \\
3-S . \text { agalactiae }\end{array}$ & Cephalexin $500 \mathrm{mg}$ QID for 30 days \\
\hline 3 & $\begin{array}{l}67 \text { years } \\
M\end{array}$ & $\begin{array}{l}\text { Left upper } \\
\text { back }\end{array}$ & Inflamed cyst & S. lugdunensis & $\begin{array}{l}\text { 1-Cefdinir } 300 \mathrm{mg} \text { BID for } 1 \text { day, then } \\
\text { 2-Doxycycline } 100 \mathrm{mg} \text { BID for } 10 \text { days }\end{array}$ \\
\hline 4 & $\begin{array}{l}80 \text { years } \\
M\end{array}$ & $\begin{array}{l}\text { Right index } \\
\text { finger nail } \\
\text { fold }\end{array}$ & Abscess & $\begin{array}{l}1-S . \text { lugdunensis } \\
2-S . \text { intermedius }\end{array}$ & $\begin{array}{l}\text { 1-Cephalexin } 500 \mathrm{mg} \text { QID for } 10 \text { days concurrent } \\
\text { with } \\
\text { 2-Sulfamethoxazole-trimethoprim } 800-160 \mathrm{mg} \\
\text { BID for } 10 \text { days }\end{array}$ \\
\hline 5 & $\begin{array}{l}82 \text { years } \\
M\end{array}$ & Left lower back & Inflamed cyst & S. lugdunensis & 1-Cephalexin $500 \mathrm{mg}$ QID daily for 30 days \\
\hline
\end{tabular}

$B I D$, twice daily; $M$, man; $m g$, milligrams; QID, four times daily; S. aureus, Staphylococcus aureus; S. lugdunensis, Staphylococcus lugdunensis; S. agalactiae, Streptococcus agalactiae; S. intermedius, Streptococcus intermedius; W, woman

${ }^{a}$ All of the sites of infection clinically presented as cellulitis. Some had an underlying infected cystic lesion, whereas others had an abscess of the nail fold

Case 2

A 30-year-old man presented for evaluation of a tender lesion on his right great toe in September 2015. There was no fever or associated systemic symptoms. His past medical history was significant for psoriasis and psoriatic arthritis. He was currently being treated with adalimumab and methotrexate.

Cutaneous examination showed erythema of the medial right great toe nail fold with an underlying abscess. A small amount of pus was expressed from the abscess and cultured. The bacterial culture grew S. lugdunensis, Staphylococcus aureus (S. aureus), and Streptococcus agalactiae ( $S$. agalactiae) as the pathogens (Table 1). Antibiotic susceptibility panels were performed (Table 2).
He was treated with incision and drainage. Empiric oral antibiotic treatment with cephalexin $500 \mathrm{mg}$, four times daily for 10 days, was initiated. Six days after starting the antibiotic, the patient mentioned that the infection had improved by $70 \%$. The cephalexin treatment was extended for another 20 days, for a total of 30 days of treatment. One month after the initiation of antibiotic therapy, the patient presented to the office for a wound check; the infection had resolved. After an additional 30 days, there were no signs of infection.

\section{Case 3}

A 67-year-old man presented for the evaluation of a tender lesion on his left upper back in February 2015. There was no fever or associated 
Table 2 Susceptibility results from bacterial cultures of patients with $S$. lugdunensis cutaneous infection

\begin{tabular}{llllll}
\hline Antibiotic & Case 1 & Case 2 & Case 3 & Case 4 & Case 5 \\
\hline Cefazolin & Susceptible & Susceptible & Susceptible & Susceptible & Susceptible \\
Clindamycin & Susceptible & Not tested & Not tested & Not tested & Not tested \\
Daptomycin & Susceptible & Susceptible & Susceptible & Susceptible & Susceptible \\
Erythromycin & Susceptible & Not tested & Not tested & Not tested & Not tested \\
Gentamicin & Susceptible & Not tested & Not tested & Not tested & Not tested \\
Linezolid & Susceptible & Susceptible & Susceptible & Susceptible & Susceptible \\
Minocycline & Susceptible & Not tested & Not tested & Not tested & Not tested \\
Moxifloxacin & Susceptible & Not tested & Not tested & Not tested & Not tested \\
Oxacillin & Susceptible & Susceptible & Susceptible & Susceptible & Susceptible \\
Penicillin G & Resistant & Resistant & Resistant & Resistant & Not tested \\
Rifampin & Susceptible & Susceptible & Susceptible & Susceptible & Susceptible \\
Tetracycline & Susceptible & Susceptible & Susceptible & Susceptible & Susceptible \\
Trimethoprim/sulfamethoxazole & Susceptible & Not tested & Not tested & Not tested & Not tested \\
Vancomycin & Susceptible & Susceptible & Susceptible & Susceptible & Susceptible \\
\hline
\end{tabular}

S. lugdunensis, Staphylococcus lugdunensis

systemic symptoms. His medical history was significant for elevated fasting glucose levels. The lesion initially appeared a few months earlier.

Cutaneous examination showed an inflamed cystic lesion with surrounding erythema on the left upper back. A small amount of pus was expressed from the lesion and cultured. Bacterial culture grew S. lugdunensis (Table 1) and antibiotic susceptibility tests were performed (Table 2).

He was treated with incision and drainage; empiric oral antibiotic treatment with cefdinir $300 \mathrm{mg}$, twice daily for 10 days, was initiated. Two days later, the patient reported that he experienced severe diarrhea. Therefore, the cefdinir was stopped and doxycycline $100 \mathrm{mg}$, twice daily for 10 days, was initiated. The infection resolved within the 10 days of treatment. There was no clinical evidence of recurrent infection 2 months after completion of treatment.

\section{Case 4}

A healthy 80-year-old man presented with right index finger pain and swelling in March 2016. There was no fever or associated systemic symptoms. His past medical history was significant for nodular basal cell carcinoma.

The patient stated that a few days prior to the presentation he was doing some gardening and scraped his finger on a plant. He subsequently developed a large blister with localized throbbing, numbness, and redness.

Cutaneous examination showed an abscess with surrounding erythema on the nail fold of the radial aspect of the right index finger. The finger was cleaned and a small amount of pus was expressed from the abscess and cultured. The bacterial culture grew $S$. lugdunensis and Streptococcus intermedius (S. intermedius) (Table 1). Antibiotic susceptibility tests were performed (Table 2). 


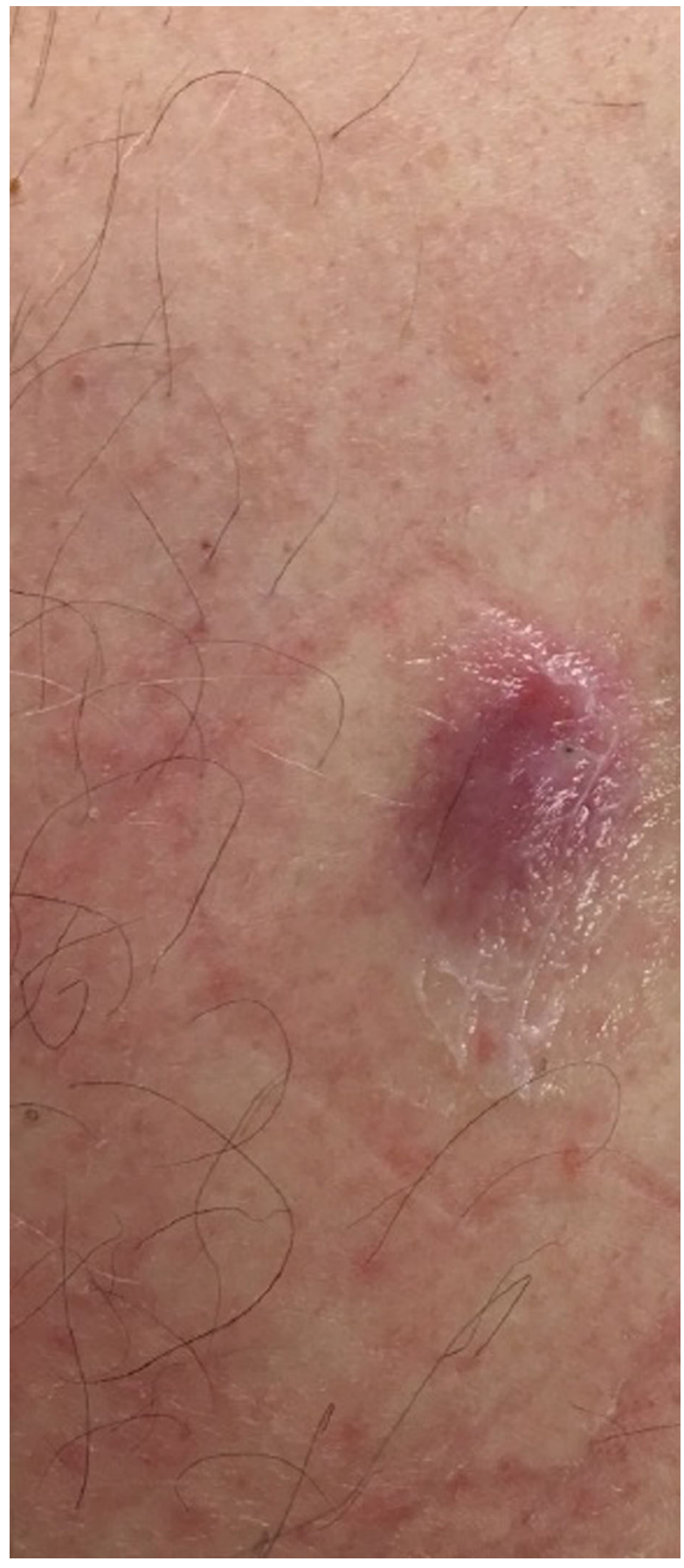

Fig. 3 Close-up view of an inflamed cystic lesion with surrounding erythema from which Staphylococcus lugdunensis was cultured on the left lower back of an 82-year-old man

He was treated with incision and drainage; empiric oral antibiotic treatment was initiated with cephalexin $500 \mathrm{mg}$, four times daily, and

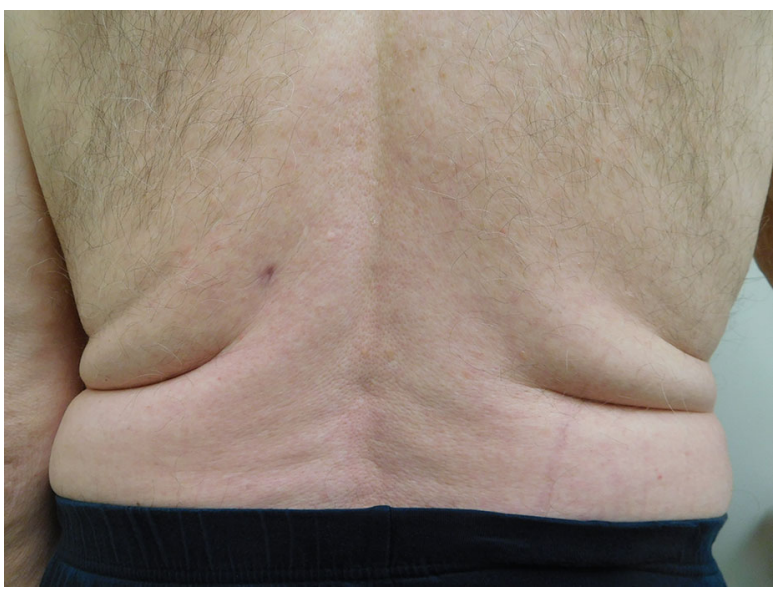

Fig. 4 Distant view of the complete resolution of the cutaneous Staphylococcus lugdunensis skin and soft tissue infection on the back of an 82-year-old man that resolved after 30 days of treatment with cephalexin $500 \mathrm{mg}$ four times daily

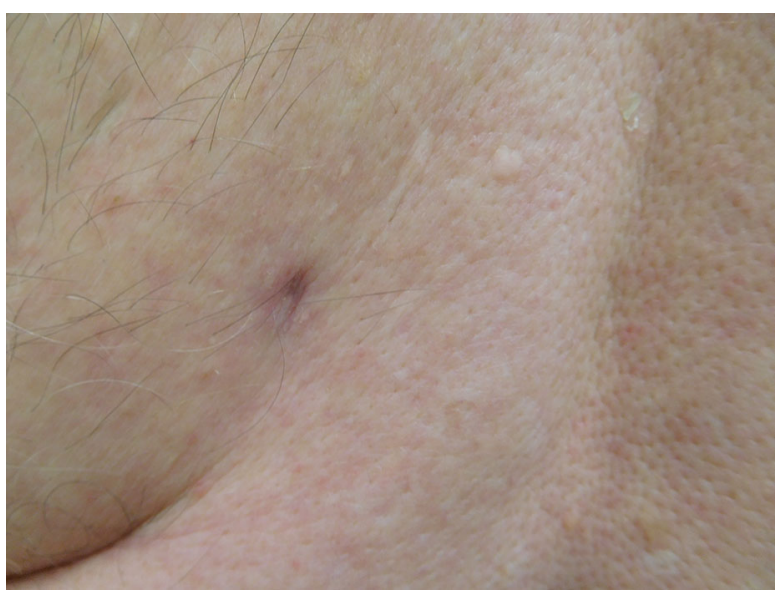

Fig. 5 Close-up view of the healed site of a Staphylococcus lugdunensis skin infection on the left lower back of an 82 -year-old man after 30 days of cephalexin $500 \mathrm{mg}$, four times daily

sulfamethoxazole-trimethoprim $800-160 \mathrm{mg}$, twice daily, for 10 days. The infection resolved within 10 days of treatment. Sixty days following completion of treatment, there were no clinical signs of recurrent infection.

\section{Case 5}

An 82-year-old man presented for evaluation of a tender lesion on his left lower back in May 
2017. There was no fever or associated systemic symptoms. His past medical history was significant for diabetes mellitus and metastatic lentigo maligna melanoma. He was being treated with pembrolizumab.

Cutaneous examination showed an inflamed cyst with surrounding erythema on the left lower back (Fig. 3). A small amount of pus from the cyst was expressed and cultured. Bacterial culture grew S. lugdunensis (Table 1) and antibiotic susceptibility tests were performed (Table 2 ).

He was treated with incision and drainage. Empiric oral antibiotic treatment with cephalexin $500 \mathrm{mg}$, four times daily for 30 days, was initiated. The infection cleared within the 30 days of the antibiotic regimen (Figs. 4, 5). There was no recurrence of the infection 60 days after completion of treatment.

Informed consent was obtained from the patients who were included in the study.

\section{DISCUSSION}

Staphylococcus organisms can be coagulase-positive or coagulase-negative. Coagulase is an enzyme produced by many bacteria that allows the conversion of prothrombin to staphylothrombin, which in turn activates the protease activity of thrombin. Activated thrombin catalyzes the conversion of fibrinogen to fibrin and other coagulation-related reactions resulting in the clotting of the blood [2, 3]. Although controversial, it has been postulated that the localized clotting elicited by coagulase can serve as a shield that protects the microorganism from the patient's immune and phagocytic defenses [3].

Coagulase-positive microorganisms tend to be considered pathogens and are traditionally associated with $S$. aureus, including methicillin-resistant and methicillin-susceptible strains. Coagulase-negative staphylococci are not able to produce coagulase and are typically considered a component of the normal flora of the skin; they are not usually considered to be pathogens.

Staphylococcus lugdunensis is a coagulase-negative staphylococcus. It was first described by Freney et al. [4]. It is typically considered part of the normal skin flora.
Recently, S. lugdunensis has been implicated as the main pathogen in a variety of infections, including central nervous system infections, endocarditis, endophthalmitis, osteomyelitis, peritonitis, prosthetic joint infections, urinary tract infections, and systemic infections $[1,5,6]$. In addition, an increasing number of the infections caused by $S$. lugdunensis are skin and soft tissue infections $[1,6,7]$.

Herchline and Ayers [7] performed a study involving 143 patients over a period of 63 months; they examined the occurrence of $S$. lugdunensis in cultures originating from 155 specimens isolated from all parts of the body. The skin and skin structures represented about 55\% of the clinical diagnosis of S. lugdunensis infections, and most of those samples originated from patients' abscesses, cellulitis, or wounds.

Five patients (one woman and four men) developed $S$. lugdunensis related-skin infections from February 19, 2015 to May 30, 2017 (Table 1). The patients' ages during the onset of the infection ranged from 30 to 82 years; the median age was 70 years. Four of the patients were older than 65 years.

The bacterial cultures from the five patients grew $S$. lugdunensis. In addition to $S$. lugdunensis, the bacterial culture from the 30-year-old man grew two other organisms: $S$. aureus and $S$. agalactiae (Streptococcus group B). Also, the bacterial culture of the 80-year-old woman grew one additional organism: $S$. intermedius. Similar to our two patients whose infections contained not only S. lugdunensis but also other organisms, Herchline and Ayers [7] noted that $60 \%$ of the cultures of specimens from their patients infected with $S$. lugdunensis also grew other microorganisms. Some of these organisms were pathogenic bacteria that can be associated with mixed infections, whereas others were bacteria that reflected the location of the lesion and included some common components of the skin flora [7].

The back was the most common location for the $S$. lugdunensis infection, followed by the digits. All of the sites of infection clinically presented as cellulitis. Some had an underlying infected cyst, whereas others had abscess of the nail fold. The lesions were incised or spontaneously ruptured. The initial clinical impression for all of the patients favored an infection 
caused by $S$. aureus, which is a prevalent pathogen in our practice. Therefore, all patients were initially treated with antibiotics that would cover methicillin-sensitive $S$. aureus. Similar to our patients, Bocher et al. [1] also observed that skin and soft tissue infections caused by $S$. lugdunensis are clinically indistinguishable from those caused by $S$. aureus.

Staphylococcus lugdunensis can also causes infection in patients with intact immunity. However, three patients were receiving immunosuppressive therapy when their infections occurred. Similarly, the studies provided by Herchline and Ayers [7] also noted that 50\% of $S$. lugdunensis infections occurred in patients who were immunosuppressed or had a prior history of trauma to the site.

The susceptibilities of the cultured strains of S. lugdunensis demonstrated that all of the organisms were sensitive to cefazolin, which is similar to observations made in patients with methicillin-sensitive $S$. aureus infections. Two patients were treated with cephalexin only. The other patients were treated with the following antibiotics: cephalexin and doxycycline or cefdinir and doxycycline or cephalexin and sulfamethoxazole-trimethoprim (Table 1). The patients were treated from 10 to 30 days until complete healing. There was no recurrence of the infection 2 months following treatment.

One limitation of our case series of $S$. lugdunenesis skin and soft tissue infections is that our methodology is prone to selection bias. The cases were retrospectively selected from among the patients evaluated by bacterial culture during a time period of 27 months. A prospective study in which all patients with suspected skin and soft tissue infections are consecutively screened for inclusion during a predefined time period could provide information on the incidence of skin and soft tissue infections of $S$. lugdunenesis as compared to those of other pathogenic bacteria.

\section{CONCLUSIONS}

Staphylococcus lugdunensis was previously considered a nonpathogenic organism and a component of the normal skin flora. However, $S$. lugdunensis can cause significant skin and soft tissue infections, and these may be more prevalent in older individuals. We observed $S$. lugdunensis skin and soft tissue infections in five individuals, four of whom were over 65 years. Three of the five patients were receiving immunosuppressive therapy. The antibiotic sensitivities of $S$. lugdunensis appear to be similar to those of methicillin-susceptible $S$. aureus. Prolonged treatment was necessary for two individuals, and therapy ranged from 10 to 30 days. In summary, this case series and relevant previous studies demonstrate that $S$. lugdunensis can cause skin and soft tissue infections which can be clinically analogous to those caused by $S$. aureus. Therefore, when $S$. lugdunensis is detected, it should be considered a potential cutaneous pathogen, especially in wounds occurring in older individuals.

\section{ACKNOWLEDGEMENTS}

No funding or sponsorship was received for this study or the publication of this article. All named authors meet the International Committee of Medical Journal Editors (ICMJE) criteria for authorship for this manuscript, take responsibility for the integrity of the work as a whole, and have given final approval of the version to be published.

Disclosures. Lucas A. Heldt Manica, BS, and Philip R. Cohen, MD, have nothing to disclose.

Compliance with Ethics Guidelines. Informed consent was obtained from the patients before they were included in the study.

Open Access. This article is distributed under the terms of the Creative Commons Attribution-NonCommercial 4.0 International License (http://creativecommons.org/licenses/ by-nc/4.0/), which permits any noncommercial use, distribution, and reproduction in any medium, provided you give appropriate credit to the original author(s) and the source, provide a link to the Creative Commons license, and indicate if changes were made. 


\section{REFERENCES}

1. Bocher S, Tonning B, Skov R, Prag J. Staphylococcus lugdunensis, a common cause of skin and soft tissue infections in the community. J Clin Microbiol. 2009;47:946-50. doi:10.1128/jcm.01024-08.

2. Cheng A, McAdow M, Kim H, Bae T, Missiakas D, Schneewind O. Contribution of coagulases towards Staphylococcus aureus disease and protective immunity. PLoS Pathog. 2010;6(8):e1001036. doi:10.1371/ journal.ppat.1001036.

3. Guo H, Hall J, Yang J, Ji Y. The SaeRS two-component system controls survival of Staphylococcus aureus in human blood through regulation of coagulase. Front Cell Infect Microbiol. 2017;7:204. doi:10.3389/fcimb. 2017.00204.

4. Freney J, Brun Y, Bes M, Meugnier H, Grimont F, Grimont P, Nervi C, Fleurette J. Staphylococcus lugdunensis sp. nov. and Staphylococcus schleiferi sp. nov., two species from human clinical specimens. Int J Syst Evol Microbiol. 1988;38:168-72. doi:10.1099/ 00207713-38-2-168.

5. Haile DT, Hughes J, Vetter E, Kohner P, Snyder R, Patel R, Cockerill FR 3rd. Frequency of isolation of Staphylococcus lugdunensis in consecutive urine cultures and relationship to urinary tract infection. J Clin Microbiol. 2002;40:654-6. doi:10.1128/jcm. 40.2.654-656.2002.

6. Frank K, Del Pozo J, Patel R. From clinical microbiology to infection pathogenesis: how daring to be different works for Staphylococcus lugdunensis. Clin Microbiol Rev. 2008;21:111-33. doi:10.1128/cmr. 00036-07.

7. Herchline T, Ayers L. Occurrence of Staphylococcus lugdunensis in consecutive clinical cultures and relationship of isolation to infection. J Clin Microbiol. 1991;29:419-21. 